\title{
CLOSED PARALLEL 1-FORMS ON INFRANILMANIFOLDS AND CALABI REDUCTIONS
}

BY

\author{
MICHAL SADOWSKI (GDAŃSK)
}

$$
\begin{aligned}
& M \text { with } \\
& \qquad \mathrm{bz}_{1}(M)=\operatorname{rankim}\left[Z\left(\pi_{1}(M)\right) \rightarrow H_{1}(M)\right]>0
\end{aligned}
$$

0. Introduction. In this paper we prove that any closed infranilmanifold

is affinely equivalent to $(F \times[0,1]) /((\alpha(x), 0) \sim(x, 1))$, where $F$ is an affine submanifold of $M$ and $\alpha: F \rightarrow F$ is an affine diffeomorphism of finite order (see Theorem 1.1 for a more precise statement). The affine equivalence is associated with every choice of an epimorphism $\varepsilon: \pi_{1}(M) \rightarrow Z$ with $\varepsilon\left(Z\left(\pi_{1}(M)\right)\right) \neq 0$ (see Theorem 3.1 below).

Theorem 4.1 is an equivariant version of Theorem 1.1. It asserts the following. If a compact Lie group $H$ acts affinely on $M$ and $\operatorname{Fix}(H ; M) \neq \varnothing$, then there is an invariant affine submanifold $F \subset M$ and an affine equivariant diffeomorphism from $F \times(0,1)$ onto $M-F$ iff $H$ fixes a nontrivial element of $Z\left(\pi_{1}(M)\right)$.

When a manifold $M$ is flat, $\mathrm{bz}_{1}(M)$ coincides with the first Betti number of $M$ (see Corollary 1.1). Hence Calabi's theorem (see, e.g., [2] and [15], Theorem 3.6.3) is a particular case of Theorem 1.1.

Theorems 1.1 and 4.1 will be derived from Proposition 2.1 describing the set $A_{\mathrm{CP}}^{\mathbf{1}}(M)$ of all closed and parallel 1 -forms on a compact infranilmanifold $M$, $\nabla$, where $\nabla$ is the canonical connection on $M$. Proposition 2.1 is a simple consequence of its particular case proved by Nomizu in [11]. Note that the corresponding result is false for higher cohomology groups (see Proposition 2.3 and [11], p. 538).

\section{The generalized Calabi reduction.}

Definition 1.1. Let $M, \nabla$ be a manifold with connection $\nabla$. Then by an affine Calabi reduction we mean an affine diffeomorphism (that is, a connection preserving diffeomorphism) $\phi: M \rightarrow F_{\alpha}, \nabla^{\prime}$, where $F$ is a manifold,

$$
F_{\alpha}=\frac{F \times[0,1]}{(\alpha(x), 0) \sim(x, 1)},
$$


$\nabla^{\prime}$ is the connection induced by a product connection on $F \times R$, and $\alpha: F \rightarrow F$ is an affine diffeomorphism of finite order $r$.

Remark 1.1. In a similar way one can define a topological Calabi reduction.

Remark 1.2. (a) An affine Calabi reduction $\phi: M \rightarrow F_{\alpha}$ determines a fibration $p: M \rightarrow S^{1}$ given by $p([x, t])=[t] \in R / Z=S^{1}$. Here $[x, t]$ is the class of $(x, t) \in F \times[0,1]$ in $F_{\alpha}$, and $[t]$ is the class of $t \in R$ in $R / Z$. $F \times S^{1}$.

(b) As $F_{\alpha^{r}}=F_{\text {id }}=F \times S^{1}$, an $r$-fold cover of $M$ is affinely diffeomorphic to

\section{DEFINITION 1.2. If}

$$
\pi: \pi_{1}(M) \rightarrow \pi_{1}(M) /\left[\pi_{1}(M), \pi_{1}(M)\right]=H_{1}(M)
$$

is the projection and $Z\left(\pi_{1}(M)\right)$ is the center of $\pi_{1}(M)$, then

$$
\mathrm{bz}_{1}(M)=\operatorname{rank} \pi\left(Z\left(\pi_{1}(M)\right)\right) \text {. }
$$

One of the basic results in the flat manifolds theory is the so-called Calabi reduction theorem. It asserts that a closed flat manifold with the first Betti number $b_{1}(M)$ greater than zero admits an affine Calabi reduction (see [2] and [15], Section 3.6; see also Corollary 1.1 below). This theorem allows us to study flat manifolds inductively using the induction on their dimensions.

Let $G$ be a nilpotent, connected, simply connected Lie group and let $\nabla_{0}$ be a connection on $G$ such that left translations in $G$ act on $T G$ as parallel translations (cf. [1], Section 7.2, and [7], Section 2). An infranilmanifold is an orbit space $M=\left(G, \nabla_{0}\right) / \Gamma$, where $\Gamma$ is a discrete group acting affinely, freely, and properly discontinuously on $G, \nabla_{0}$ and such that $G \cap \Gamma$ has finite index in $\Gamma$. We will always assume that $G \cap \Gamma$ acts on $G$ by right translations. An infranilmanifold $G / \Gamma$ is a flat manifold if $G$ is isomorphic to $R^{n}$.

A closed manifold $M(\operatorname{dim} M>4)$ is homeomorphic to an infranilmanifold iff $\pi_{1}(M)$ is a finite extension of a nilpotent, torsion free, finitely generated group and $\pi_{i}(M)=0$ for $i \geqslant 2$ (see [6], Theorem 5.1).

It is easy to verify that one-parameter subgroups of $G$ are exactly geodesics and $f \in \operatorname{Aff}(G)$ (where $\operatorname{Aff}(G)$ denotes the group of all affine diffeomorphisms of $G$ ) if and only if $f=L_{\theta} \circ A$, where $g \in G, L_{g}(y)=g y$ for $y \in G$, and $A$ is an automorphism of $G$ (cf. [7], Section 2). Hence affine submanifolds of $G$, $\nabla$ are exactly algebraically affine submanifolds of $G$. By an algebraically affine submanifold of $G$ we mean a submanifold $L_{h}(H) \subset G$, where $H$ is a Lie subgroup of $G$ and $h \in G$.

The following result generalizes Calabi reduction to infranilmanifolds.

THEOREM 1.1. Let $M, \nabla$ be a closed infranilmanifold with a flat canonical connection $\nabla$. Then there is an affine Calabi reduction of $M$ if and only if $\mathrm{bz}_{1}(M)>0$. 
Remark 1.3. (a) It is easy to see that the existence of a topological Calabi reduction implies $b_{1}(M)>0$. So we should prove only the converse implication.

(b) The fact that the existence of a topological Calabi fibration implies the existence of an affine Calabi fibration seems to be a very special property of infranilmanifolds. It can fail even for nonpositive curvature manifolds. To be more specific, for every closed manifold $M$ admitting a metric of nonpositive curvature and satisfying $Z\left(\pi_{1}(M)\right) \neq\{1\}$ there is (see Theorem 3.1 in [5] and [9]) a topological Calabi fibration but not always there is an affine Calabi fibration. See [9], p. 222, for a counterexample.

(c) The fact that $\phi: M \rightarrow F_{\alpha}$ is a Calabi reduction of an infranilmanifold $M$ means that $F$ is covered by an algebraically affine submanifold $\tilde{F}=L_{h}(H)$ (where $H$ is a Lie subgroup of $G$ and $h \in H$ ) of $G, G$ is isomorphic to $H \times R$, and $\phi$ is covered by an algebraically affine diffeomorphism $\tilde{\phi}: G \rightarrow G$. Hence Theorem 1.1 can be treated as a purely algebraic result.

(d) A topological (or smooth) version of Theorem 1.1 can be found in [5] (Theorem 3.1) and [10] (Section 4.7, Corollary 1). In [10], Section 4.7, topological Calabi fibrations are treated as a particular case of a more general notion of a Seifert fiber space with typical fiber $S^{1}$ and Corollary 1 there follows from a more general result ([10], Section 4.7, Theorem 1).

COROLlaRY 1.1. If $M$ is a closed riemannian flat manifold, then $\mathrm{bz}_{1}(M)=\mathrm{b}_{1}(M)$. Hence Calabi's theorem is a particular case of Theorem 1.1.

Proof. The proof of Corollary 1.1 is implicitly described in the proof of the Calabi reduction (see, e.g., [15], Section 3.6). For convenience of the reader we present here a short direct argument. The flatness of $M$ implies that harmonic forms on $M$ are parallel. Every parallel 1-form $\omega$ determines a parallel vector field $X_{\omega}$ that is perpendicular to $\operatorname{ker} \omega$ and satisfies $\omega\left(X_{\omega}(x)\right)=1$ for $x \in M$. It is well known that $I_{0}(M)$, the identity component of the isometry group of a closed flat manifold $M$, is generated by parallel fields. Since $I_{0}(M)$ is a compact, commutative (as parallel fields commute) Lie group, $I_{0}(M)$ is a torus $T^{k}$ and our argument shows that $k \geqslant b_{1}(M)$. In particular, there is an $S^{1}$-action $\phi_{t}: M \rightarrow M, t \in[0,1]$, generated by a parallel vector field $X$.

It is well known (see [4], Lemma 4.2) that every orbit of our $S^{1}$-action determines a central element of $\pi_{1}(M)$. As the vector field $X$ is parallel, the 1 -form $\omega$, given by $\omega(v)=\langle X, v\rangle$, is parallel and, in particular, it is harmonic. For a fixed orbit $c:[0,1] \rightarrow M, c(t)=\phi_{t}(x)$, we have

$$
\int_{c} \omega=\int_{0}^{1} \omega(d c / d t) d t=\int_{0}^{1} \omega(X) d t=|X|^{2}>0 .
$$

This holds for any $S^{1}$-action embedded into our $T^{k}$-action. Hence we have a monomorphism

$$
\pi_{1}\left(T^{k}\right) \stackrel{e v_{*}}{\longrightarrow} H_{1}(M) \rightarrow Z^{k}
$$


where $\mathrm{ev}_{*}$ is induced by ev: $T^{k} \rightarrow M, \operatorname{ev}(t)=t x_{0}$ (here $x_{0}$ is a chosen point of $M)$, and where the second map is induced by the projection onto the quotient of $H_{1}(M)$ by its torsion subgroup. As

$$
\operatorname{imev}_{*} \subset \operatorname{im}\left[Z\left(\pi_{1}(M)\right) \rightarrow H_{1}(M)\right],
$$

we have $\mathrm{bz}_{1}(M) \geqslant k$. This completes the proof of Corollary 1.1.

EXAMPLE 1.1. The last statement, that $b_{1}(M)=b z_{1}(M)$ for a flat manifold $M$, is false for infranilmanifolds. A well-known counterexample is the following. Let $G$ be the Heisenberg group, i.e., the group of real $3 \times 3$ upper triangular matrices with diagonal entries equal to 1 . It is the simplest noncommutative nilpotent Lie group. Let $\Gamma$ be its subgroup of all matrices with integer entries and let $M=G / \Gamma$. Then $G$ is diffeomorphic to $R^{3}, \Gamma \approx \pi_{1}(M)$, and it is easy to see that $Z(\Gamma)=[\Gamma, \Gamma] \approx Z$. Hence $\mathrm{bz}_{1}(M)=0$. However,

$$
H_{1}(M) \approx \Gamma /[\Gamma, \Gamma] \approx Z \oplus Z \quad \text { and } \quad b_{1}(M)=2 .
$$

2. Closed parallel 1-forms on infranilmanifolds. In this section we prove the following

Proposition 2.1. Let $M=G / \Gamma$ be a closed infranilmanifold and let $\nabla$ be a canonical connection on $M$. Then every cohomology class $v \in H^{1}(M ; R)$ contains a unique $\nabla$-parallel 1-form. In particular, $\operatorname{dim} A_{\mathrm{CP}}^{1}(M)=\mathrm{b}_{1}(M)$.

Throughout this section the following notation will be used. By $L(G)$ we denote the Lie algebra of a Lie group $G$. If $H$ is a group (or if $H$ is a Lie algebra), then $H^{\mathrm{ab}}$ denotes its abelianization, i.e., $H^{\mathrm{ab}}=H /[H, H]$, and $Z(H)$ stands for the center of $H$. By $B^{k}(G)$ we denote the set of all bi-invariant $k$-forms on a Lie group $G$, and by $\pi^{*}: B^{k}\left(G^{\text {ab }}\right) \rightarrow B^{k}(G)$ the homomorphism induced by the canonical projection $\pi: G \rightarrow G^{\mathrm{ab}}$. The set of all parallel and closed $k$-forms on an infranilmanifold $M, \nabla$ (where $\nabla$ is a canonical connection) will be denoted by $A_{\mathrm{CP}}^{k}(M)$.

We will need some known facts (cf. [11], Section 4, and [12], Appendix 2) concerning closed and left-invariant 1 -forms on Lie groups. They can be stated as follows.

Proposition 2.2. (a) Let $G$ be a connected Lie group and let $\omega$ be a left-invariant 1-form on $G$. The following conditions are equivalent:

(i) the form $\omega$ is bi-invariant;

(ii) the form $\omega$ is closed;

(iii) the homomorphism $L(G) \rightarrow R \approx L(R)$ determined by $\omega$ is a Lie algebra homomorphism.

(b) The homomorphism $\pi^{*}: B^{1}\left(G^{\mathrm{ab}}\right) \rightarrow B^{1}(G)$ is an isomorphism.

The implication (i) $\Rightarrow$ (ii) is known (see, e.g., [12], Appendix 2, Section 2). The equivalence (ii) $\Leftrightarrow$ (iii) follows immediately from the formula $2 d \omega(X, Y)$ $=\omega([X, Y])$, where $X, Y \in L(G)$. If (iii) holds, then $\omega: L(G) \rightarrow L(R)$ has a unique 
factorization $\omega=\omega_{0} \circ P$, where $P: L(G) \rightarrow L\left(G^{\mathrm{ab}}\right)$ is the canonical projection and $\omega_{0}: L\left(G^{\mathrm{ab}}\right) \rightarrow L(R)$. Note that $P=d \pi$ and $\omega=\pi^{*} \omega_{0}$. Since the form $\omega_{0}$ is bi-invariant, the form $\omega$ is bi-invariant as well.

Corollary 2.1 (see [13], Corollary 7.28, and [11]). If $M=G / \Gamma$ is a closed nilmanifold, then

$$
\operatorname{dim} A_{\mathrm{CP}}^{1}(M)=\operatorname{dim} B^{1}(G)=\operatorname{dim} G^{\mathrm{ab}}=\operatorname{rank} \Gamma^{\mathrm{ab}}=\operatorname{rank} \pi_{1}(M)^{\mathrm{ab}}=\mathrm{b}_{1}(M) .
$$

Proof. Every 1-form $\omega \in A_{\mathrm{CP}}^{\mathbf{1}}(M)$ is covered by a bi-invariant 1-form and every bi-invariant 1 -form can be projected onto $M$. Hence $\operatorname{dim} A_{\mathrm{CP}}^{1}(M)$ $=\operatorname{dim} G^{\mathrm{ab}}$. Now it suffices to check that $\operatorname{dim} G^{\mathrm{ab}}=\operatorname{rank} \Gamma^{\mathrm{ab}}$. This is well known (see [13], Theorem 2.1).

Proof of Proposition 2.1. The manifold $M$ can be written as the orbit space $\hat{M} / A$, where $\hat{M}$ is a nilmanifold and $A$ is a finite group acting affinely and freely on $\hat{M}$. Let $q: \hat{M} \rightarrow M$ be the canonical projection. By Theorem 1 of [11] (and by Proposition 2.2) the cohomology class $q^{*} v$ is represented by a unique form $\hat{\omega} \in A_{\mathrm{CP}}^{1}(\hat{M})$.

It suffices to show that the form $\hat{\omega}$ is $A$-invariant. In order to prove this note that the cohomology class $q^{*} v$ is $A$-invariant. Consider

$$
\hat{\eta}=(1 /|A|) \sum_{a \in A} a^{*} \hat{\omega}
$$

As $A \subseteq \operatorname{Aff}(M)$, we have $\hat{\eta} \in A_{\mathrm{CP}}^{1}(\hat{M})$. As $\hat{\eta}$ and $\hat{\omega}$ belong to the same cohomology class, $\hat{\eta}=\hat{\omega}$. The homomorphism $q^{*}: H^{1}(M ; R) \rightarrow H^{1}(\hat{M} ; R)$ is a monomorphism, because the covering $q$ is finite. If $\omega, \omega_{1} \in A_{\mathrm{CP}}^{1}(M) \cap v$, then, by Theorem 1 of [11] again, $q^{*} \omega=q^{*} \omega_{1}$, so that $\omega=\omega_{1}$. This proves the proposition.

Remark 2.1. If an infranilmanifold $M$ is a closed flat manifold, then harmonic forms on $M$ are parallel. Hence, by the Hodge theorem, any cohomology class $v \in H^{*}(M ; R)$ contains a unique parallel form. This cannot be extended even to nilmanifolds (cf. [11], p. 538).

Proposition 2.3. Let $M=H / \Gamma$ be the Heisenberg manifold (see Example 1.1). Then $A_{\mathrm{CP}}^{2}(M)$ is a one-dimensional vector space. All forms belonging to $A_{\mathrm{CP}}^{2}(M)$ are exact.

Proof. The Lie algebra $R(H)$ of right-invariant vector fields is generated by the vector fields $X, Y, Z$ such that $[X, Z]=[Y, Z]=0,[X, Y]=Z$. The field $Z$ is bi-invariant. For any right-invariant 1 -form $\eta$ and for any $U, V \in R(H)$ we have $2 d \eta(U, V)=\eta([U, V])$. Hence the form $d \eta$ is bi-invariant. It determines a 2 -form $\omega \in A_{\mathrm{CP}}^{1}(M)$. This form is exact, because a right-invariant form $\eta$ can be projected onto $M$ (the group $\Gamma$ acts on $H$ by right translations).

The fact that $\operatorname{dim} A_{\mathrm{CP}}^{2}(M)=1$ follows from the results of [11] (see [11], p. 538, and the proof of Theorem 2 there). The proof of Proposition 2.3 is complete. 
3. Proof of Theorem 1.1. Let $M$ be a closed infranilmanifold with $\mathrm{bz}_{1}(M)>0$, let $\pi: \pi_{1}(M) \rightarrow H_{1}(M)$ be the canonical projection, and let $\sigma \in Z\left(\pi_{1}(M)\right)-\{1\}$ be such that $\pi(\sigma) \neq 0$. Our proof of Theorem 1.1 is based on the existence of an affine $S^{1}$-action $\phi_{t}: M \rightarrow M, t \in[0,1]$, whose orbit containing the base point belongs to $\sigma$ (see [8], Section 4.3). We show that for any epimorphism $h: \pi_{1}(M) \rightarrow \pi_{1}\left(S^{1}\right)$ satisfying $h(\sigma) \neq 0$ there is an affine $S^{1}$-equivariant Calabi fibration $p: M \rightarrow S^{1}$ such that $p_{*}=h$. Here by an $S^{1}$-equivariant fibration we mean a fibration $p$ such that $p\left(\phi_{t}(x)\right)-p(x)$ depends on $t$ only.

Theorem 1.1 is a particular case of the following more technical result:

THEOREM 3.1. Let $M=G / \Gamma$ be a closed infranilmanifold, let $\sigma \in Z\left(\pi_{1}(M)\right)$, and let an $S^{1}$-action $\phi_{t}: M \rightarrow M, t \in[0,1]$, be as above. Let $h: \pi_{1}(M) \rightarrow \pi_{1}\left(S^{1}\right) \approx Z$ be any epimorphism such that $h(\sigma)=r \in Z-\{0\}$, let $\Delta=\operatorname{ker} h, N=G / \Delta$, and let $\bar{\phi}_{t}: N \rightarrow N, t \in R$, denote the $R$-action covering our $S^{1}$-action on $M$. Then there are an affine submanifold $V \subset M$ and an affine diffeomorphism $\alpha: V \rightarrow V$ such that

(a) the mapping $\psi: V \times \boldsymbol{R} \rightarrow N$ given by $\psi(u, t)=\dot{\bar{\phi}}_{t}(u)$ is an $\boldsymbol{R}$-equivariant affine diffeomorphism;

(b) the group of covering transformations of the covering $q: N \rightarrow M$ is infinite cyclic, and if $\delta$ denotes its generator, then, under the above identification of $V \times \boldsymbol{R}$ with $N$,

$$
\delta(u, t)=(\alpha(u), t+1 / r)
$$

(c) $\alpha^{r}=\mathrm{id}_{V}$;

(d) the fibration $p: M \rightarrow S^{1}$ determined by our twisted decomposition $M=(V \times R) /\langle\delta\rangle=V_{\alpha}$ (see Remark 1.2 (a)) satisfies $p_{*}=h$; its typical fiber is affinely diffeomorphic to $V$.

Remark 3.1. A smooth variant of Theorem 3.1 (valid for all closed manifolds and for all smooth homologically injective $S^{1}$-actions) can be found in [5], Theorem 4.2.

Proof of Theorem 3.1. The homomorphism $h$ determines a homomorphism $h_{0} \in \operatorname{Hom}\left(H_{1}(M ; Z) ; Z\right)$ and $h_{0}$ determines an element

$$
[h] \in \operatorname{im}\left[H^{1}(M ; Z) \rightarrow H^{1}(M ; R)\right]
$$

characterized by the equality

$$
\int_{\gamma}[h]=h(\gamma) \quad \text { for } \gamma \in \pi_{1}(M)
$$

By Proposition 2.1 there is a unique parallel 1-form $\omega$ representing [h].

The form $\omega$ is nonvanishing and has integral periods. Fix $x_{0} \in M$. By [3], the map $p: M \rightarrow R / Z=S^{1}$ given by

$$
p(x)=\int_{x_{0}}^{x} \omega(\bmod Z)
$$


is a well-defined fibration over $S^{1}$ such that the leaves of the foliation tangent to $\operatorname{ker} \omega$ are connected components of the fibers of $p$. The form $\omega$ is $\phi_{t}$-invariant, because $\phi_{t}^{*} \omega$ is a parallel form cohomologous to $\omega$ (cf. Proposition 2.1). It follows that the fibration $p$ is $S^{1}$-equivariant.

Let $X$ be the vector field generating the $S^{1}$-action. Assume that $X(x) \in \operatorname{ker} \omega$ for some $x \in M$. Note that $\operatorname{ker} \omega$ is the bundle tangent to the fibers of $p$. Consider $c:[0,1] \rightarrow M$ defined by $c(t)=\phi_{t}(x)$. Then $c \in \sigma$ and $(d c / d t)(t)$ $=X(c(t))$. By (1) and by the $S^{1}$-invariance of $X$ and $\omega$ we have

$$
0 \neq h(\sigma)=\int_{c} \omega=\int_{0}^{1} \omega(X) d t=\omega(X(x))=0 .
$$

This contradiction shows that the fibers of $p$ are transversal to the orbits of the $S^{1}$-action. Note that

$$
\begin{aligned}
p\left(\phi_{t}(x)\right)-p(x) & =\int_{x}^{\phi_{t}(x)} \omega(\bmod Z)=\int_{0}^{t} \omega(X) d t(\bmod Z) \\
& =t \omega(X(x))(\bmod Z)=\operatorname{th}(\sigma)(\bmod Z)=\operatorname{tr}(\bmod Z) .
\end{aligned}
$$

Hence

$$
\begin{gathered}
p\left(\phi_{t}(x)\right)-p(x)=\operatorname{tr}(\bmod Z), \\
p_{*}(\sigma)=h(\sigma) .
\end{gathered}
$$

Let $V$ be a fiber of $p$. By (3) every fiber of $p$ can be written as $\phi_{t}(V)$ for some $t \in[0,1]$. The field $X$ is parallel (because $\phi_{t}(x)=x g_{t}=g_{t} x$, where $t \rightarrow g_{t}$ is a one-parameter subgroup of the center $Z(G)$; see [8], Section 4.3) and the bundle $\operatorname{ker} \omega$ is parallel. Hence it is not difficult to show that for any fiber $\phi_{s}(V)$ and for some $\varepsilon>0$ the map

$$
\Phi: V \times(-\varepsilon, \varepsilon) \rightarrow\left\{\phi_{t}(V): t \in(s-\varepsilon, s+\varepsilon)\right\},
$$

given by $\Phi(u, t)=\phi_{t+s}(u)$, is an affine diffeomorphism. Under this identification, $p$ can be written as $p(u, t)=r(t+s)$. It follows that the map $p$ is affine, as claimed.

Let $q$ : $N \rightarrow M$ be the canonical projection, let $\bar{p}: N \rightarrow R^{k}$ be the fibration covering $p$, let $V=\bar{p}^{-1}(0)$, and $u \in V$. By (3),

$$
\bar{p}\left(\bar{\phi}_{t}(y)\right)=t r+\bar{p}(y) \quad \text { for } y \in N .
$$

Hence $\bar{p}^{-1}(s)=\bar{\phi}_{s / r}(V)$ for $s \in R$. It is clear that $\psi: V \times R \rightarrow V$, given by $\psi(v, t)=\bar{\phi}_{t}(v)$, is an $R$-equivariant diffeomorphism. The diffeomorphism $\psi$ is affine (compare the argument showing that $\Phi$ is affine).

The homomorphism $h_{1}: \Gamma / \Delta \rightarrow Z$ determined by $h$ is an isomorphism, because $h$ is an epimorphism and $\Delta=\operatorname{ker} h$. Here $\Delta$ is canonically identified with the corresponding subgroup of $\Gamma$. It follows that $\Gamma / \Delta$ is generated by $\delta=h_{1}^{-1}(1)$. Let $\sigma_{0}$ be the image of $\sigma \in \pi_{1}(M) \approx \Gamma$ in $\Gamma / \Delta$. 
Let $\tilde{\phi}_{t}: G \rightarrow G, t \in R$, be the $R$-action covering the $S^{1}$-action. Under the canonical identification of $\pi_{1}\left(M, x_{0}\right)$ with $\Gamma, \tilde{\phi}_{1}$ corresponds to $\sigma$ so that $\sigma_{0}=\bar{\phi}_{1}$. Recall that $\gamma \in \Gamma$ is identified with the homotopy class of $P \circ c$, where $c:[0,1] \rightarrow M$ is any curve joining $x_{0}$ to $\gamma\left(x_{0}\right)$, and $P$ is the canonical projection of the universal covering space of $M$ onto $M$. As $p_{*}(\sigma)=h(\sigma)=r$, we have $\sigma_{0}=\delta^{r}$.

The equality $\delta=h^{-1}(1)$ implies that $\bar{p}(\delta(u))=\bar{p}(u)+1$ for $u \in V$. Since $\bar{p}\left(\bar{\phi}_{t}(u)\right)=r t$, we have $\delta(V)=\bar{\phi}_{1 / r}(V)$. Let $\alpha=\bar{\phi}_{1 / r}^{-1} \circ \delta$. Then $\alpha(V)=V$. As $\bar{\phi}_{0}=\operatorname{id}_{N}$ and $\bar{\phi}_{t}$ covers $\phi_{t}: M \rightarrow M$, it follows that $\bar{\phi}_{t}$ commutes with $\delta$ for every $t \in R$. Hence $\alpha \circ \bar{\phi}_{t}=\bar{\phi}_{t} \circ \alpha$ and $\alpha^{r}=$ id.

Under the identification $\psi: V \times \boldsymbol{R} \rightarrow N$, the diffeomorphism $\alpha$ can be written as $\alpha(u, t)=(\alpha(u), t)$. As $M=N /\langle\delta\rangle$, it follows that $\left.q\right|_{V}$ carries $V$ diffeomorphically onto a fiber of $p$, which proves (b). The group $\Delta \times\langle\sigma\rangle$ is a subgroup of $\Gamma$ of index $r$ because $\Delta \times\langle\sigma\rangle=h^{-1}(r Z)$. As $h=p_{*}$ on $\Delta \times\langle\sigma\rangle$, it follows that $p_{*}=h$. This completes the proof of Theorem 3.1.

4. Equivariant affine Calabi reductions. Our aim here is to generalize the results of the previous sections to the equivariant case. Let $H$ be a Lie group acting on a manifold $V$ and let $\alpha: V \rightarrow V$ be an $H$-equivariant diffeomorphism. Let $[x, t]$ denote the class of $(x, t) \in V \times[0,1]$ in $V_{\alpha}$. For every $a \in \pi_{1}(M)$ the symbol $\varrho(a)$ denotes the image of $a$ in $H_{1}(M, Q)$.

Definition 4.1. The manifold $V_{\alpha}$ with the action of $H$ given by. $h[x, t]=[h(x), t]$ is called the equivariant mapping torus of $\alpha$.

THEOREM 4.1. Let $H$ be a compact Lie group acting affinely on a closed infranilmanifold $M=G / \Gamma$ with a fixed point $*$. Then the following conditions are equivalent:

(i) The manifold $M$ is $H$-equivariantly and affinely diffeomorphic to a mapping torus $V_{\alpha}$ of an $H$-equivariant, periodic, affine diffeomorphism $\alpha: V \rightarrow V$.

(ii) There is $\sigma \in Z\left(\pi_{1}(M)\right)$ such that $\varrho(\sigma) \neq 0$ and $h_{*}(\sigma)=\sigma$ for $h \in H$.

Remark 4.1. The case where the manifold $M$ is flat is easier (cf. [14], Section 1).

Proof. Assume that (i) holds. Let $r$ be the order of $\alpha$. Then $\phi_{t}: M \rightarrow M$, $t \in[0,1]$, given by $\phi_{t}([x, s])=[x, s+r t]$, where $[x, s]$ is treated as the class of $(x, s) \in V \times R$ in $V_{\alpha}$, is an $S^{1}$-action on $M$. Let $\sigma \in \pi_{1}(M)$ be the class of the orbit of our fixed point *. Then we know ([4], Lemma 4.2) that $\sigma \in Z\left(\pi_{1}(M)\right)$. As an $r$-fold cover of $M$ is $V_{\text {id }}=V \times S^{1}$, we have $\varrho(\sigma) \neq 0$. By Definition 4.1 the action of $H$ on the orbit of $*$ is trivial.

Assume (ii). Let $\phi_{t}: M \rightarrow M, t \in[0,1]$, be the parallel $S^{1}$-action whose orbits belong to $\sigma$ (see Section 3). Since $\varrho(\sigma) \neq 0$, there is an epimorphism $\mu: \pi_{1}(M) \rightarrow Z$ such that $\mu(\sigma) \neq 0$. The identity component $H_{0}$ of $H$ acts trivially on $\pi_{1}(M, *)$ and $K \cdot=H / H_{0}$ is a finite group. Set

$$
\lambda(\gamma)=\sum_{k \in K} \mu\left(k_{*}(\gamma)\right) \quad \text { for } \gamma \in \pi_{1}(M) .
$$

The homomorphism $\lambda$ is $H$-equivariant and $\lambda(\sigma)=|K| \mu(\sigma) \neq 0$. 
Take $[\lambda] \in \operatorname{iin}\left[H^{1}(M ; Z) \rightarrow H^{1}(M ; R)\right]$ corresponding to $\lambda$ (see Section 3) and a parallel form $\omega$ representing [ $\lambda]$ (see Proposition 2.1). By the $H$-invariance of [ $\lambda]$ and by the uniqueness of $\omega$, the form $\omega$ is $H$-invariant. It follows (compare the proof of Theorem 3.1) that the corresponding $S^{1}$-equivariant affine fibration $p: M \rightarrow S^{1}$ is $H$-equivariant.

By [8], Section 4.3, we have $\tilde{\phi}_{t}(x)=g_{t} x=x g_{t}$, where $\tilde{\phi}_{t}: G \rightarrow G, t \in R$, is the action of $R$ covering the $S^{1}$-action and $t \rightarrow g_{t}$ is a one-parameter subgroup of $Z(G)$. Let $h \in H$. Take $\tilde{h} \in \operatorname{Aff}(G)$ covering $h$. Let $\tilde{h}=L_{u} \circ \Phi$, where $u \in G$, $\Phi \in \operatorname{Aut}(G)$, and let $b_{t}=\Phi\left(g_{t}\right)$. Since $H$ acts trivially on $\sigma$, the affine actions

$$
\phi_{t}: M \rightarrow M, \quad \psi_{t}=h \circ \phi_{t} \circ h^{-1}: M \rightarrow M, \quad t \in[0,1],
$$

have homotopic orbits so that $\tilde{\phi}_{1}=\tilde{\psi}_{1}$. A direct calculation yields

$$
\tilde{\psi}_{t}(x)=\left(\tilde{h} \circ \tilde{\phi}_{t} \circ \tilde{h}^{-1}\right)(x)=\Phi\left(g_{t}\right) x=b_{t} x,
$$

and as $\tilde{\phi}_{1}=\tilde{\psi}_{1}$, we have $b_{1}=g_{1}$. It follows that $b_{t}=g_{t}$ for $t \in[0,1]$, because $b_{t}, g_{t} \in Z(G)$. Hence $\tilde{\psi}_{t}(x)=g_{t} x=\tilde{\phi}_{t}(x)$ for every $x \in G$. Thus the $S^{1}$-action $\phi_{t}$ commutes with $h$ and $h\left(\phi_{t}(*)\right)=\psi_{t}(*)=\phi_{t}(*)$.

Let $F=p^{-1}(0)$. As $* \in F$, we have $h(F)=F$ for $h \in H$. If $x \in M, h \in H$, then $x \in \phi_{t}(F)$ for some $t \in[0,1], p(h x)=p\left(h \phi_{t}(*)\right)=p\left(\phi_{t}(*)\right)=p(x)$, and Theorem 4.1 follows from Theorem 3.1.

\section{REFERENCES}

[1] P. Buser and H. Karcher, Gromov's Almost Flat Manifolds, Astérisque 81, Société Mathématique de France, 1981.

[2] E. Calabi, Closed, locally euclidean, 4-dimensional manifolds, Bull. Amer. Math. Soc. 63 (1957), p. 135.

[3] J. Ce rf, 1-formes fermées non singulières sur les variétés compactes de dimension 3, pp. 205-219 in: Bourbaki Seminar, Vol. 1980/81, Lecture Notes in Math. 901, Springer, Berlin-New York 1981.

[4] P. E. Conner and F. R a y mond, Actions of compact Lie groups on a spherical manifolds, pp. 227-264 in: Topology of Manifolds (Proc. Inst., Univ. of Georgia, Athens, Ga., 1969), Markham, Chicago 1970.

[5] - Injective operations of the toral groups, Topology 10 (1971), pp. 283-296.

[6] F. T. Farrell and W. C. Hsiang, Topological characterisation of flat and almost flat Riemannian manifolds $M^{n}(n \neq 3,4)$, Amer. J. Math. 105 (1983), pp. 641-673.

[7] F. Ka mber and P. Tonde ur; Flat Manifolds, Lecture Notes in Math. 67, Springer, Berlin 1980.

[8] Y. Kamishima, K. B. Lee and F. Raymond, The Seifert construction and its applications to infranilmanifolds, Quart. J. Math. Oxford Ser. (2) 34 (1983), pp. 433-452.

[9] H. B. Lawson and S. T. Yau, Compact manifolds of nonpositive curvature, J. Differential Geom. 7 (1972), pp. 211-228.

[10] K. B. Lee and F. Raymond, The role of Seifert fiber spaces in transformation groups, pp. 367-425 in: Group Actions on Manifolds (Boulder, Colo., 1983), Contemp. Math. 36, Amer. Math. Soc., Providence,. R.I., 1985.

[11] K. Nomizu, On the cohomology of compact homogeneous spaces of nilpotent Lie groups, Ann. of Math. (2) 59 (1954), pp. 531-538. 
[12] H. V. Pittie, Characteristic Classes of Foliations, Pitman, London 1976.

[13] M. S. Raghunathan, Discrete Subgroups of Lie Groups, Springer, Berlin 1972.

[14] M. Sadowski, Calabi's reductions and centrally trivial actions, to appear.

[15]-J. Wolf, Spaces of Constant Curvature, McGraw Hill, New York 1967.

DEPARTMENT OF MATHEMATICS

THE UNIVERSITY OF GDAŃSK

80-952 GDAŃSK

WITA STWOSZA 57, POLAND

Reçu par la Rédaction le 5.10.1988;

en version modifiée le 114.1989 